21.0. The p-value of 0.05 was consider statistically significant. Results: There were 562 cases recruited in the study. Mean age of patients was 43 years. The mean BMI was $25.74 \mathrm{~kg} / \mathrm{m}^{2}$ (range, 14.38-66.12). The BMI was divided into 3 groups: 1 ) Normal weight (BMI, 18.5-22.9 kg/m²) was 210 cases (37.3\%); 2) Over-weight (BMI, 23-24.9 kg/m²) was 90 cases (16.0\%); and 3) Obese (BMI $\geq 25 \mathrm{~kg} / \mathrm{m}^{2}$ ) was 262 cases $(46.6 \%)$. The pathological reports demonstrated 36 cases of endometrial hyperplasia and 22 cases of endometrial cancer. There was a correlation between obese women and endometrial hyperplasia with odds ratio 5.9 and p-value 0.015 . However, there were no statistically significant between other BMI patterns and endometrial pathology.

Conclusion: There was a significant correlation between obesity and endometrial hyperplasia in premenopausal women with abnormal uterine bleeding.

Poster (E23)

Endometrial Hyperplasia, Endometrial Intra-epithelial Neoplasia, and Endometrial Cancer

https://doi.org/10.3802/jgo.2021.32.S1.E23

\section{Incidence of parametrial spreading in endometrial cancer at stages I and II in Siriraj Hospital}

\section{Suwanit Therasakvichya, "Sasimas Rujirawong, Boonlert Viriyapak, Atthapon Jaishuen}

Faculty of Medicine, Siriraj Hospital, Mahidol University, Bangkok, Thailand (suwanit.the@mahidol.ac.th)

Objective: To identify the incidence of parametrial involvement in endometrial cancer stages I and II and the related risk factors. Methods: The retrospective descriptive study was performed to review data from patients with endometrial cancer, clinical stages I and II who underwent surgical treatment as primary therapy. The data included patients' status, pathological reports (especially parametrial involvement), stage, adjuvant treatment, complications of treatment, and response of disease.

Results: The medical charts of 76 patients were reviewed and 3 cases were excluded from this study because of incomplete pathological report. The mean age was 58 years old. Most of them were early stage (IA, IB and II that defined according to International Federation of Gynecology and Obstetrics 2009 surgical stage with radical or modified radical hysterectomy), 31 (42.5\%) tumor grade I, and $76.7 \%$ endometrioid adenocarcinoma. Three patients (4.1\%) had parametrial involvement. The tumor spread into parametrium by lymphovascular space invasion in 2 cases and one case had lymph node metastasis. Because of the small number of cases, it is hard to use this data to identify the risk factors and correlation of parametrial involvement.

Conclusion: The incidence of parametrial involvement was too small. The benefit of radical or modified radical hysterectomy to identify parametrial involvement in endometrial cancer is not strongly suggested from the results of this study. A prospective study with adequate sample size and long term follow up should be considered.

Poster (E24)

Endometrial Hyperplasia, Endometrial Intra-epithelial Neoplasia, and Endometrial Cancer

https://doi.org/10.3802/jgo.2021.32.S1.E24

\section{Management of inoperable endometrial cancer}

\section{Supakorn Pitakkarnkul,' Saranya Chanpanitkitchot, ${ }^{2}$ Siriwan Tangjitgamol ${ }^{3, *}$}

'Gynecologic Oncology Division, National Cancer Institute, Bangkok, Thailand

${ }^{2}$ Department of Obstetrics and Gynecology, Rajavithi Hospital, College of Medicine, Rangsit University, Bangkok, Thailand ${ }^{3}$ Department of Obstetrics and Gynecology, Faculty of Medicine Vajira Hospital, Navamindradhiraj University, Bangkok, Thailand (siriwanonco@ yahoo.com)

Objective: Most existing information about treatment for inoperable epithelial-myoepithelial carcinoma (EMC) are derived from retrospective studies or case series. Without evidence-based data from randomized study, collective data from available reports would be helpful for a gynecologic oncologist to select an alternative treatment option for EMC patients who are not candidates for primary surgery.

Methods: This work collected and summarized data from literature review of relevant studies according to the treatment intention (definitive or neoadjuvant). Treatment given with a palliative aim, either chemo- or hormonal therapy, or best supportive care is beyond the scope of this review.

Results: The 3 major types of treatment for inoperable EMC were radiation therapy, chemotherapy, or their combination, and interval surgery. Radiation therapy alone (of different modes) was used as a sole definitive modality of therapy particularly for early-stage diseases which limited to uterine body, cervix, with or without parametrial invasion. A more common treatment modality was neoadjuvant treatment prior to surgery. Post-operative adjuvant treatment was also sometimes used depending mainly on the sites of disease and results of surgery. Data of neoadjuvant hormonal or radiation therapy were limited focusing on laboratory outcomes or having only small number of patients, respectively. Most neoadjuvant treatment before surgery was chemotherapy and 